\title{
Realizing skilled companionship in nursing: A utopian idea or difficult challenge?
}

\section{Abstract}

Aim: The question being considered in this discussion paper is whether nurses' practice can be usefully characterized in terms of skilled companionship.

Background: A nurse's role might be characterized as one of a "skilled companion," a concept that brings together the scientific and moral basis of nursing practice. It is precisely this focus on integrating both "skill" and "companionship" characteristics of nurses' practice that largely determines their effectiveness as care providers and their specific contribution to health-care outcomes.

Method: Discursive paper that invites readers to explore and arrive at a more comprehensive understanding of nurses' daily practice, one from an ethical perspective that is based on empirical data from the study of patients' caring experiences, nurses' caring experiences, and nurses' ethical practice in daily care.

Conclusion: Research in this era of health care highlights an important dilemma that the nursing profession is confronted with daily: realizing nurses' role as a skilled companion in an environment where this concept of nursing care is discouraged, or even thwarted. Although the ethical dimension is explicitly and universally recognized as a core dimension of nursing care, research clearly highlights nurses' difficulties in translating this element into daily practice. Most disturbing in these findings is that both patients and nurses feel compelled to reach a compromise in order to "survive" in this often-chaotic caring environment.

Relevance to clinical practice: It appears today that nurses' intrinsic strengths and potential are largely underutilized in daily practice. More than ever, the nursing profession is 
challenged to reflect on the future position, aspirations, and responsibilities of nurses, to make clear choices, and to act accordingly. Viewing and implementing the nurse's role as one of a skilled companion promise better health-care delivery in a postmodern world.

Keywords: Nursing ethics, nursing care, patient experiences, nursing experiences, ethical practice 


\section{Realizing skilled companionship in nursing: A utopian idea or difficult challenge?}

\section{AIMS \& BACKGROUND}

Caring for a patient in the postmodern world is a real challenge for nurses. We live in an era in which change is so rapid and dramatic, unlike any period before. Thus, the past is of little help in aiding us to deal with actual and likely future challenges in health care. Societal evolution and health-care reforms have created a culture of uncertainty, which is ubiquitous and unavoidable in clinical practice (Seely, 2013). Mainly because patients are increasingly informed and often have multiple comorbidities, and because of globalization across many domains and enhanced use of technology, nurses more than ever before are confronted with complex and unpredictable issues, as well as unanswerable questions (Vaismoradi et al. 2011). Regardless, they are expected to autonomously make decisions and deliver "good care." "Good care" refers to the promotion of the patient as a human being in all his or her dimensions; i.e., considering improving a patient's physical, psychological, relational, social, moral, and spiritual well-being (Gastmans et al. 1998). It is this emphasis on human flourishing that turns nursing care into an ethical enterprise. Whatever nurses do or decide must always be related to promoting the patient's well-being by providing "good care" in the broadest sense of the word.

This kind of care obviously does not only require medical and technical competence, but also the nurse's ability to genuinely "care" for the patient. Caring behavior requires the integration of virtue and expert activity, both considered as main components in realizing 'good care' (Gastmans et al., 1998).The caring encounter between nurse and patient is the starting point in good nursing care and requires that the nurse and the patient deal successfully and effectively with illness, vulnerability, and suffering (Gastmans et al., 1998). 
Through this relationship, nurses are encouraged to transcend their normal nursing routine and "bend" the rules, norms, and procedures in order to find a personal and effective solution to their patients' problems and needs. It is precisely in this relational context through a process of choice and deliberation- that difficult choices are made, priorities are established and consensus is reached about the effective caring answers to patient's illness (Gastmans et al., 1998). The nurse as well as the patient are both involved in this process of choice and deliberation, each from his or her particular life context.

In this context, the nurses' role might be characterized as one of a "skilled companion," a concept that brings together the scientific and moral basis of nursing practice (Kitson 1996, Tichen 2000, O'Connell 2008). This metaphor incorporates both the lived or actual experience of illness (values) and the scientific knowledge (facts) in every aspect of nursing care (O'Connel 2008). Realizing this companionship model requires from nurses commitment, mutuality, and a virtually constant presence (Titchen 2000). Achieving the full capacity of companionship lies in being able to discover the uniqueness of a person, to sense his or her needs, and to really help this person. Understanding patients' real world from their perspective seems to make their vulnerability more bearable, or can even transform it into strength (Gjengedal et al. 2013). A good understanding of the patient's illness experience is therefore seen as an essential pre-condition for good care (Shell \& O'Hare 2013). In conclusion, specifically the focus on the integration of both the "skills" and "companionship" aspects largely determines nurses' effectiveness and specific contribution to health-care outcomes (Kitson 1996, Pearson et al. 1997, Komatsu \& Yagasaki 2014). 
Realizing skilled companionship in a high-tech, rational, and productive care system appears as a real challenge (Kieft et al. 2014). Nurses are incited to navigate a course leading them beyond a purely biomedical framework into a postmodern paradigm that focuses on the patient's personal well-being (Brown et al. 2009). The invisibility of many parts of the caring practice makes this aspect of care often subordinate to other more visible activities, particularly in a society that does not value caring (Corbin 2008, Maben 2008). Over the last decades we have seen a progressive idealization of putting into practice technical, intellectual, and scientific advances of nursing-all valuable elements in the professionalization of nursing. However, this is accompanied by a devaluation of the ethical aspects of nursing. Yet, it is precisely the integration of both components - scientific and ethical components-that determine the essence of nursing and explains why nurses can make a clear difference in the experiences of patients. Nursing seems to have turned into a disintegrated profession, one having difficulties in realizing its full potential (Reed 2000, Turkel et al. 2012).

This paper invites the reader to more deeply consider and understand nurses' daily practice from an ethical perspective. Can we accurately and genuinely describe nurses' practice as one of skilled companionship? How do nurses really "care" for patients in daily practice? To what extent does the patient and his/her illness experiences occupy a central place in a nurse's actual practice? This exploration is informed by our own and other researchers' empirical data about nurses' practice, using three avenues: the perspective of the patients, the view of nurses, and nurses' ethical reasoning and practice in ethically sensitive situations. A critical review of the literature in these three research areas have been performed. We specifically focused on empirical studies exploring (1) patients' 
experiences of care and care encounters, (2) nurses' stories and perceptions on care, and (3) the way nurses reason and practice in ethically difficult situations. For the last mentioned research track we used the systematic literature review of Goethals et al (2010).

\section{NURSING CARE FROM AN ETHICAL PERSPECTIVE}

\section{Patients' caring experiences}

Exploring patients' experiences of care provides an interesting way to understand what patients consider essential within care and how these quality aspects are evaluated (Rademakers et al. 2011). The increasing attention given to patient-reported outcomes has given rise to a growing number of qualitative studies aimed at better understanding the patient's perspective of care. A Belgian study that investigated the impact of a clinical leadership program on nurses and patient outcomes (Dierckx de Casterlé et al. 2002, De Geest et al. 2003) revealed some interesting findings in this area. A careful analysis of patients' experiences revealed that different levels of care, in terms of quality, are given. The nature of the relationship between patients and nurses seemed to be a significant determinant of the level of care experienced. Three levels could be discerned.

First-level care reflects a rather instrumental, functional relationship between patients and nurses (Dierckx de Casterlé et al. 2002). The focus of this level of care revolves around "providing care" in answer to only the physical and medical needs of patients. The patients' stories revealed that the nurses act routinely and take little account of a patient as a unique individual. In short, the nurses do what they have to do and what they are asked to do, but nothing more. They try to create an environment wherein patients can survive and 
feel safe. At this level, the patient is viewed as a passive agent. The patient naturally accepts the action of "the expert" and often plays the role of "a good patient." The researchers concluded that care providing at this level could be considered as "minimal" or "basic" care (Dierckx de Casterlé et al. 2002).

Second-level care is characterized by actions and an attitude that clearly recognizes the patient and his or her individual care needs. The nurses act together with the patient and do more than the bare essentials. According to the patients, nurses performing at this level anticipate their needs and aim to take these into account in providing care; they help the patients understand their situation and how to cope with it. They creatively and critically look for the best caring solution, one that mainly derives from the perspective of an expert. The relationship between patient and nurse is a professional one, but patients underscored the asymmetric character of this relationship. The authors labeled this level as "professional" care (Dierckx de Casterlé et al. 2002).

Care comprising the third level is driven by the concept of "skilled companionship" (Tichen 2000). Patients reported that they felt accompanied and supported by the empathic presence of a nurse while being sick, treated, or rehabilitated. The nurse accompanies the patient in the role as a professional and as a person willing to express her/his vulnerability. This companionship is based on a high level of competency. The harmonious integration of competence and a caring attitude is the key characteristic of third-level care. Knowing and considering patients' experiences and needs are the starting point of the nurses' care; their personal well-being is the nurses' main focus. More than at the other two levels at this third level of care, nurses aim to discover what is important for patients, how patients experience their illness and treatment, and how they, as professionals and as people, can support 
patients in this process. At this level, the relationship between a patient and a nurse is somewhat symmetric, characterized by mutual trust and equality. Patients feel that nurses understand what they are going through and what this means for them. In short, nurses are physically, emotionally, and existentially close to the patient.

Although this level of care is strongly desired implicitly or even expected, patients rarely experience level-three care in practice. Level-one and level-two care were most frequently represented in the patients' stories. Patients did not complain, however, and mostly attributed the lack of level-three care as being due to the difficult caring context, more specifically to the lack of nurses' available time, to difficulties in realizing teamwork among the care staff, and the lack of a prominent caring culture (Dierckx de Casterlé et al. 2002).

Qualitative studies related to patients' experiences of care revealed empirical support for these above-mentioned conclusions. Patients reported a continuum of quality in care, ranging from very poor to high, with high quality care referring to situations in which both competency and a caring attitude are present (Attree 2001, Bridges et al. 2010, Izumi et al. 2010). The interactional and interpersonal aspects of care appeared to be a central issue in the way quality was evaluated from a patient's perspective (Attree 2001, Larrabee et al. 2004, Davis 2005, Radwin et al. 2005, Sorlie et al. 2006, Kutney-Lee et al. 2009, Wyson \& Driver 2009, Bridges et al. 2010). Patients looked for authentic encounters with nurses, ones involving simply their presence, recognition of the patients' issues, their availability, and mutuality (Wyson \& Driver 2009, Bridges et al. 2010). However, they often felt left alone and abandoned (Holopainen et al. 2012). According to patients, it was precisely the lack of interactional aspects of care that caused them to feel isolated and invisible (Moran 
2008). The interactional aspects of care appeared to become even more important when patients' vulnerability increased (Izumi et al. 2010, Scott et al. 2013).

The literature surprisingly reports that patients, in general, were satisfied, even though the care they received was not optimal (Attree 2001, Sorlie et al. 2006, Nayeri \& Agjahana 2010). Patients related information about factors that they did not consider as being optimal, but which they explained as being compromises they had to accept as being a necessary part of their stay in the ward (Shattell 2002, Walker 2002, Sorlie et al. 2006, Nayeri \& Agjahana 2010). Yet, patients' compromising may be viewed as a response to the threat they feel to their integrity (Irurita \& Williams 2000, Shattell 2002).

In summary, exploring patients' experiences of care revealed that those aspects that are considered as essential within care and could be characterized as comprising skilled companionship are often missed in daily care.

\section{Nurses' caring experiences}

Investigating nurses' perspective on caring practice is another way to better understand the nature of the daily care nurses deliver. The above-mentioned study of Dierckx de Casterlé et al. (2002) also explored nurses' experiences of care by using self-reported case studies. Nurses were asked to describe a recent care situation they had with a patient that they believed qualified as an example of good nursing practice, and to describe why they evaluated this particular instance positively. The study revealed that nurses also perceive their mission as one of a skilled companion. In the words of the nurses, they said they 
"wanted to be there for the patient, with all their competence," trying their best to translate their competence in a caring relationship. However, their stories also revealed that, in reality, nurses often experienced nursing care more coldly, in essence as a medicaltechnical care product. The relational aspects of care were limited to a rather asymmetric relationship, wherein nurses offered patients their expertise. Mutuality and equity were mostly absent. Most efforts were targeted at providing professional, individualized care, one focusing on the patient and his/her disease. Technical competence and anticipation of potential complications were considered to be essential. It is as if they felt obliged to limit their care to a "lower level," in the vernacular of Dierckx de Casterlé et al. (2002). Their message was clear: In the actual nursing context, there was no, or hardly any, room for realizing care that could be characterized as comprising skilled companionship (Dierckx de Casterlé et al. 2002).

These findings are in agreement with the study of Maben et al. (2007), in which they described nurses' ideals in terms of high-quality, patient-centered, holistic, and evidencebased care. At the same time, the nurse respondents described how the caring aspects were undermined and undervalued in favor of more task-oriented and visible aspects of their work. The difference between how nurses would ideally like to care for patients and the realities they face in practice is also supported by other studies (e.g., West et al. 2005, Siebens et al. 2006, Murphy 2007, Wallerstedt \& Anderstedt 2007, Frilund et al. 2013). A nationwide Belgian study about nurses' professional self-image (Siebens et al. 2006) showed that nurses perceived their most important contributions to be in the field of medicaltechnical care. The Belgian nurses believed that nurses' actual care is primarily focused on curing patients and on detecting problems and potential complications in order to provide 
clinically effective and safe care. "Caring" aspects are considered to be fundamental to good practice, but in day-to-day practice they played only a minor role, according to these nurses.

Insufficient time, resources, limited collaboration and support from managers, and a lack of a "patient-centered" culture were found to be the main reasons for nurses' dissatisfaction (Williams 1998, Varcoe et al. 2004, West et al. 2005, Siebens et al. 2006, Wallerstedt \& Anderstedt 2007, Maben 2008, Frilund et al. 2013). The majority of West et al.'s (2005) respondents felt overworked and reported that they lacked sufficient time to perform essential nursing tasks, such as addressing patients' anxieties, fears, and concerns. Siebens et al.'s (2006) respondents reported a lack of adequate time and support to integrate their medical-technical competence into a caring relationship. This concern was also expressed in Williams' study (1998), in which nurses dealing with time constraints used a process termed, "selective focusing." The nurses narrowly focused on certain patients, or on certain patient needs, in order to be better able to deliver quality care. The nurses' choices and priorities were guided by patient safety, implying that physical needs were given priority over psychosocial needs. Also disquieting in this context is nurses' experience that it is virtually impossible to deliver care that follows one's own ethical values or to even discuss ethical problems in team meetings (Siebens et al. 2006, Frilund et al. 2013). These findings are consistent with those of Varcoe et al. (2004), who referred to nurses' complaints about the lack of support and leadership for carrying out ethically based practice. The organizational focus on cost-efficiency resulted in routinized and task-oriented care giving, which left nurses feeling pressured to increase productivity rather than quality (Maben 2008, Kieft et al. 2014). 
Above mentioned studies revealed that many nurses were worried about virtually being unable to provide the level of care they wished to provide, resulting in patients' needs and wishes remaining unaddressed and nurses' growing dissatisfaction in daily practice. These results might explain the high degree of tension, frustration, moral distress, and burnout reported in many nursing studies (e.g., Varcoe et al. 2004, Corley et al. 2005, Edward \& Hercelinskyj 2007, Maben 2008, Aiken et al. 2011, McHugh et al. 2011,). In summary, the results of these studies bring to the fore problems in the nursing work environment that impede rather than support nurses in realizing good nursing caring, one which comprises skilled companionship. A "poor working environment" has clearly been associated with negative outcomes for nurses and quality of care (Aiken et al. 2011).

\section{Nurses' ethical practice}

Empirical research on nurses' ethical practice is a third way to explore nurses' daily practice from an ethical perspective. A meta-analysis of nurses' ethical practice $(n=1592$ registered nurses) was performed using empirical data from nine studies (Dierckx de Casterlé et al. 2008). The findings of this study revealed a strong tendency of nurses to adopt a conformist ethical practice. Confronted with daily ethical dilemmas, nurses were mainly guided by contextual factors rather than by patients' personal needs and well-being. More concretely, prevailing views and expectations, guidelines, rules or norms, orders of physicians, procedures or protocols, among others, largely determined how nurses reasoned and practiced in ethically sensitive care situations. The results strongly suggested that nurses simply conformed to the requirements of the environment, without critically questioning them in view of good care. It should be noted that conformist practice by 
definition rules out a critical and creative reflection in light of the best caring answer, resulting in nursing care that is mainly determined by the environment instead of what is relevant and needed for the individual patient (Dierckx de Casterlé et al. 2008).

Actually, guidelines (such as recommendations for handling medical prescriptions and protocols of a care unit) are important to nursing, because they provide a professional framework that supports nurses in daily care practice. However, these guidelines remain tools in the search of good care and should never be regarded as an end in themselves. Guidelines need to be critically evaluated in each individual patient situation and in light of patient well-being (Dierckx de Casterlé et al. 2008). Failing to reflect critically on daily practice seriously decreases the possibilities to promote the patient's personal well-being. Taking into account the complexity of each care situation and the uniqueness of each patient, it is doubtful that high-quality care can be achieved if nurses adopt a conformist practice. The greater diversity in a patient's experiences, needs, and reactions, the smaller the probability that conformist care will contribute to the patient's well-being. Good care requires nurses to be prepared to adjust rules, norms, and other conventions if they do not fit a patient's situation, and then to act according to their own analysis and values. In other words, nurses need to adapt their practice in ways that are most meaningful to a patient's well-being (Dierckx de Casterlé et al 2008). From an ethical point of view, nurses' tendency to adopt a conformist practice is worrisome and works against achieving a skilled companionship role.

A systematic review of the literature on nurses' ethical practice provides support for the notion of nurses' sometimes adopting conformist practice (Goethals et al. 2010). This review revealed that environmental factors have a large impact on nurses' ethical practice. 
Confronted with different values and expectations (e.g., of patients and their family, colleagues, physicians, and others), in addition to rules, routines, procedures, and guidelines that are specific to the ward or institution, nurses experience many difficulties that hinder their personal decision-making process. Several researchers demonstrated how difficult working conditions caused nurses to conform to prevailing expectations and views, resulting in less individually tailored care (e.g., Rodney et al. 2002, Varcoe et al. 2004, Kim et al. 2007). Nurses' tendency to conform to others' expectations and views, together with difficult working conditions, appear to discourage nurses from acting ethically in practice. However, the Goethals et al. (2010) review also revealed that some nurses are capable of overcoming the conformist approach to nursing practice. Possession of certain personal qualities, such as competency, readiness to take risks, boldness, and strong problem-solving skills seem to stimulate nurses to critically search in creative ways for the most effective caring answer, rather than to acquiesce and conform to environmental demands (Goethals et al. 2010).

\section{DISCUSSION}

Exploration of examples of nursing care from an ethical perspective gives rise to some concern. Although the ethical dimension is still recognized as the core element of nursing care, research clearly points to nurses' difficulties in translating all aspects of this into daily care practice. Viewing nursing care as "skilled companionship" is an implicit wish of patients as well as nurses. Nurses really desire "to be there" for their patients, to understand their illness experience, and to have a positive impact on their quality of life. A recent Canadian study highlights young nurses' desire to make a difference in people's lives. 
Altruism, caring, and compassion were revealed to be central themes in the analysis of nurses' choices for choosing nursing as career (Price et al. 2013). Nurses' values-driven ambition reflects the intrinsic power of the nursing profession, which allows nurses to really make a positive difference in health care. However, nurses today struggle in their job to put into practice these essential nursing values. It seems as if this intrinsic strength of nurses and their potential are largely underutilized in daily practice. This thwarting effectively defeats achieving the delivery of nursing care in terms of skilled companionship.

Nurses' tendency to acquiesce to conformism, especially in ethically sensitive situations, is alarming but at the same time hardly surprising in the current state of the health-care system. Faced with the growing complexity and the lack of certainty, understanding, and predictability - all characteristics of the postmodern era-all human beings tend to look for something to "hold onto" in order to reduce anxiety associated with uncertainty and insecurity and to be able to survive this complicated world. Conformist practice is a convenient way to function in and deal with these circumstances. Rules, norms, prescriptions, and protocols offer a welcome way for nurses to simplify and control complex situations, leading to a false sense of feeling safety. Not surprising, adoption of this approach is often desired across other domains of this postmodern world. Seely (2013) described this tendency as a paradoxical response to uncertainty. Although the inevitable presence of uncertainty and its pseudo benefits are ubiquitous in health care, we continually seek to reduce and control it. By accepting this intrinsic uncertainty, health-care providers could improve their outlook toward health-care delivery (Seely 2013). This view helps us to understand why providing ethical care requires nurses to display not only competence and compassion, but also courage. Entering into a caring relationship that 
guides patients through a journey of uncertainty and then acting according to one's ethical values and convictions, even when this means going against current expectations or conventions, are real challenges for nurses (Thorup et al. 2011, Komatsu \& Yagasaki 2014).

Patients, more than ever, are looking for health-care providers that are able to understand what they are going through and how their illness affects their life and their well-being. Lack of certainty and security enhances the need of human beings, most certainly vulnerable persons, to be understood and accompanied. Within the current highly technical, rational, fragmented, and rather chaotic health-care environment, there is an urgent appeal from patients, especially the chronically ill, to receive a more holistic, individualized, and human-oriented kind of care. Research, as well as practice, supports the hypothesis that this kind of nursing care is often absent.

Most disturbing in these findings is that both patients and nurses feel compelled to reach a compromise in good care in order to "survive" in a difficult caring environment. Nurses' most important challenge seems to be the realization of skilled companionship within a caring environment, in which there is barely or no room for a skilled companionship kind of care. This dilemma results in a struggle that often leads to care that nurses are not proud of but which they consider as "acceptable," given the circumstances. This is an alarming finding, because we know that being unable to incorporate one's own values into daily practice may result in moral distress (Gutierrez 2005, Goethals et al. 2010) and in questioning one's self-identity (Doane et al. 2004). Nurses often respond to moral distress by being less involved with patients and family and by providing less individualized care, which leads to a vicious cycle of more distress and less acceptable care (Gutierrez 2005). This feeling of moral distress may lead to more job dissatisfaction for nurses, staff turnover, 
health problems, burnout, and even nurses' decision to leave the profession (Gutierrez 2005, Goethals et al. 2010).

Patients, too, express their willingness to accept "compromised" or "second-class" care, one that mainly focuses on coldly alleviating only their physical health problems. Patients' apparent "satisfaction" with nursing care might be considered to be their way of trying to preserve their self-esteem and autonomy (Shattell 2004 ). Sorlie et al. (2006) rightly warned care providers of the risk of losing the focus on patients' vulnerability when accepting consciously or unconsciously the patients' compromises. Patients experience vulnerability in many ways, resulting in feelings of insecurity (Sorlie et al. 2006). The way nurses interact with patients may increase or decrease the patients' feeling of vulnerability (Gjengedal et al. 2013). Being sensitive to patients' vulnerability appears to be a key to achieving an ethically based practice. This requires an understanding of patients' real-world situation, as seen through the patients' eyes. This underscores the importance of nurses' having courage to enter into the patient-nurse relationship and their ability to recognize the patients' vulnerability and to help them face these experiences (Thorup et al. 2011, Gjengedal et al. 2013).

\section{RELEVANCE TO CLINICAL PRACTICE:}

In summary, exploration of nurses' practice from an ethical perspective has revealed an important dilemma that confronts the nursing profession today: How do nurses realize skilled companionship in an environment where there seems to be little room for this concept of nursing care? It is a challenge for nurses to deal with this dilemma. Uncertainty and ambivalence, besides routinization and mechanization, make it difficult for nurses to 
establish and maintain relationships, contributing specifically to failure to care (Rendall \& Mckeown 2013).

Although the impact of nurses' specific work context on ethical practice cannot be denied, the failure of caring in nursing cannot be justified or fully explained by contextual factors alone. The current trajectory of societal and health-care evolution forces the nursing profession to think deeply about the future position, aspirations, and responsibilities of nurses. Some authors explicitly point to the urgent need to explicitly articulate the value of caring in the nursing profession (Corbin 2008, Griffiths 2008, Holopainen et al. 2012, Turkel et al. 2012). Do our nursing profession actually esteem caring tasks-tasks some might consider to be of minor importance-and really believe in the absolute necessity of these encounters to connect with ill persons and to really make a difference in health care?

Shifting the focus away from a mainly technical, intellectual, and scientific model that currently dominates postmodern health care is a challenging goal for educators, care providers, and leaders (Bridges \& Meyer 2010, Milton 2011). Educators are challenged to create a context in which a caring attitude can be meaningfully taught and cultivated (Vanlaere \& Gastmans 2007). Part of this context is helping students to recognize, understand, and respond to the suffering of others (Rolfe \& Gardner 2014). Facilitating students' and practitioners' critical self-reflection represents a key component of successful ethical education (Vanlaere \& Gastmans 2007, Levesque et al. 2013,). In clinical practice, we need role models, ones able to reflect critically on daily practice and to harmoniously integrate technical and caring skills. Role models are crucial in creating environments conducive to caring and making caring "visible" (Maben 2008). Promoting a caring ethos in a busy, high-tech, and rational work environment comes to the fore as one of the most crucial 
abilities nurses need to develop and practice (Maben 2008). This underscores the importance of having strong clinical leaders who are committed and able to create the culture and context in which skilled companionship can flourish (Bridges et al. 2010). Nursing leaders are in a position to advocate for the essential values of nursing and to find out how best to use the opportunities and strengths of the actual health-care context to promote these values, and in this way maximize nurses' strengths and potential in practice.

In conclusion, if we consider that "skilled companionship" in nursing might be applied as a metaphor, then our professional challenge is not only to demonstrate how advance nursing practice can be cost- and clinically effective in the arena of health care, but also to advocate how the broader implementation of this view of caring can be a moral force for positive change in society as a whole (Kitson 1996).

\section{References}

Aiken, LH, Sloane, DM, Clarke, S, Poghosyan, L, Cho, E, You, L, Finlayson, M, Kanai-Pak, M \& Aungsuroch, Y (2011) Importance of work environments on hospital outcomes in nine countries. International Journal of Quality Health Care 23(4), 357-364.

Attree, M (2001) Patients' and relatives' experiences and perspectives of 'Good' and 'Not so Good' quality care. Journal of Advanced Nursing 33(4), 456-466.

Bridges, J, Flatley, M \& Meyer, J (2010) Older people's and relatives' experiences in acute care settings: systematic review and synthesis of qualitative studies . International Journal of Nursing Studies 47, 89-107.

Brown, CA, Bannigan, K \& Gill, JR (2009) Questionning: A critical skill in postmodern healthcare service delivery. Australian Occupational Therapy Journal 56, 206-210. 
Corbin, J (2008) Is caring a lost art in nursing? International Journal of Nursing Studies 45, 163-165.

Corley, MC, Minick, P, Elswick, R K, Jacobs, M (2005) Nurse moral distress and ethical work environment. Nursing Ethics 12(4), 381-90.

Davis, LA (2005) A phenomenological study of patient expectations concerning nursing care. Holistic Nursing Practice 19,126-133.

De Geest, S, Claessens, P, Longerich, H Schubert, M A the International Clinical Leadership Group (2003) Transformational Leadership: Worthwile the investment. European Journal of Cardiovascular Nursing 2(1), 3-5.

Dierckx de Casterlé, B, Izumi, S, Godfrey, NS \& Denhaerynck (2008) Nurses' responses to ethical dilemmas in nursing practice: meta-analysis. Journal of Advanced Nursing 63(6), 540-549.

Dierckx de Casterlé, B, De Geest, S, Darras, E, Stordeur, S \& the International Leadership Group (2002) Clinical Leadership Development Project: an intervention study to enhance transformational leadership style of head nurses in the realm of skilled companionship. Not published Research Report, Ministry of Social Welfare, Public Health and Life Environment, Belgium.

Doane, G, Pauly B, Brown, H \& McPherson, G (2004) Exploring the heart of ethical nursing practice: implications for ethics education. Nursing Ethics 11(3), 240-253.

Edward, K \& Hercelinskyj, G (2007) Burnout in the caring nurse: learning resilient behaviours. Britisch Journal of Nursing 16(4):240-2.

Frilund, M, Eriksson, K \& Fagerström, L (2013) The caregivers' possibilities of providing ethically good care for older people - a study on caregovers' ethical approach. Nordic College of Caring Science 28, 245-254.

Gastmans, C, Dierckx de Casterlé, B, Schotsman, P (1998) Nursing considered as moral practice: a philosophical-ethical interpretation of nursing. Kennedy Institute of Ethics Journal 8(1), 43-69. 
Gjengedal, E, Ekra, E M, Hol, H, Kjelsvik, M, Lykkeslet, E, Michaelsen, R, Orøy, A, Skrondal, T, Sundal, H, Solfrid, V \& Wogn-Henriksen, K (2013) Vulnerability in healthcare - reflections on encounters in every day practice. Nursing Philosophy 14, 127-138

Goethals, S, Gastmans, C, Dierckx de Casterlé, B (2010) Nurses' ethical reasoning and behaviour: a literature review. International Journal of Nursing Studies 47(5), 635-650.

Griffiths, P (2008) The art of losing ...? A response to the question 'is caring a lost art?' International Journal of Nursing Studie 45, 329-332.

Guteriez, KM (2005) Critical care nurses' perceptions of and responses to moral distress. Dimensions of Critical Care Nursing 24(5), 229-241.

Holopainem, G, Kasén, A \& Nyström, L (2012) The space of togetherness - a caring encounter. Scandinavian Journal of Caring Sciences 28(1), 186-92.

Irurita, VF \& Willams, AM (2000) Balancing and compromising: nurses and patients preserving integrity of self and each other. International Journal of Nursing Studies 5, 579589.

Izumi, S, Baggs, J G \& Knafl, K A (2010) Quality nursing care for hospitalized patients with advanced illness: concept development. Research in Nursing \& Health 33, 299-315.

Kieft, RA, de Brouwer, BB, Francke, AL \& Delnoij, DM (2014) How nurses and their work environment affect patient experiences of the quality of care: a qualitative study. $B M C$ Health Services Research 14: 249 doi: 10 1186/1472-6963-14-249.

Kim, YS, Park, JH \& Han, SS (2007) Differences in moral judgment between nursing students and qualified nurses. Nursing Ethics 14(3), 303-319.

Kitson, A (1996) Does nursing have a future? British Journal of Medicine 313,1647-1651.

Komatsu, H \& Yagasaki, K (2014) The power of nursing: Guiding patients through a journey of uncertainty. European Journal of Oncology NursingEuropean Journal of Oncology Nursing $18,419-424$.

Kutney-Lee, A, McHugh, MD, Sloane, DM et al (2009) Nursing: a key to patient satisfaction. Health Affairs 28 (4), w669-w677. 
Larrabee, JH, Ostrow, CL, Withrow, ML, Janney, MA, Hobbs, GR \& Burant, C (2004) Predictors of patient satisfaction with inpatient hospital nursing care. Research in Nursing \& Health 27, 254-268.

Levesque, MC, Hovey, RB \& Bedos, C (2013) Advancing patient-centered care through transformative educational leadership: a critical review of health care professional preparation for patient-centered care. Journal of Healthcare Leadership 5, 35-46.

Maben, J (2008) The art of caring: Invisible and subordinated? A response to Juliet Corbin: 'Is caring a lost art in nursing?' International Journal of Nursing Studies 45, 335-338.

Maben, J, Latter, S, Mcleod Clark, J (2007) The challenges of maintaining ideals and standards in professional practice: evidence form a longitudinal qualitative study. Nursing Inquiry 14(2), 99-113.

McHugh, MD, Kutney-Lee, A Cimiotti, JP, Sloan, DM \& Aiken, LH (2011) Nurses' widespread job dissatisfaction, burnout, and frustration with health benefits signal problems for patient care. Health Affairs 30, 2, 202-210.

Milton, CL (2011) An ethical exploration of quality and safety initiatives in nurse practice. Nursing Science Quaterly 24 (2), 107-110.

Murphy, K (2007) A qualitative study explaining nurses' perceptions of quality care for older people in long-term care settings in Ireland. Journal of Clinical Nursing 16, 477-485. Moran, A (2008) The lived experience of hemodialysis patients: treatment and quality of life. Unpublished PhD Thesis, Dublin City University, Dublin http://doras dcu ie/558/2/Aoife_MoranPhD2008 pdf.

Nayeri, ND \& Agjahana, M (2010) Patients' privacy and satisfaction in the emergent department: a descriptive analytical study. Nursing Ethics 17, 167-176.

O'Connell, E (2008) Therapeutic relationships in critical care nursing: a reflection on practice. Nursing in Critical Care 13(3), 138-143. 
Pearson, A, Borbasi S \& Walsh, K (1997) Practicing nursing therapeutically through acting as a skilled companion on the illness journey. Advanced Practice Nursing Quaterly/Summer $3(1), 46-52$.

Price, SL, Hall, LM, Angus, JE \& Peter, E (2013) Choosing nursing as a career: a narrative analysis of millennial nurses' career choice of virtue. Nursing inquiry 20(4), 305-316.

Rademakers, J, Delnoij, D \& de Boer, D (2011) Structure, process or outcome: which contributes most to patients' overall assessment of healthcare quality? BMJ Quality \& Safety $20,326-331$

Radwin, LE, Farquhar, SL, Knowles, MN \& Virchick, BG (2005) Cancer patients' descriptions of their nursing care. Journal of Advanced Nursing 50(2), 162-169.

Randall, D \& McKeown, M (2013) Editorial: Failure to care: nursing in a state of liquid modernity. Journal of Clinical Nursing 23, 766-767

Reed, P (2000) Nursing reformation: Historical reflections and philosophical foundations. Nursing Science Quaterly 20, 308-311.

Rodney, P, Varcoe, C, Storch, JL, Mcpherson, G, Mahoney, K, Browns, H, Paylu, B, Hartrick, G \& starzomski, R (2009) Navigating towards a moral horizon: a multisite qualitative study of ethical practice in nursing. Canadian Journal of Nuring Research 41(1), 292-319.

Rolfe, G \& Gardner, LD (2014) The compassion deficit and what we do about it: a response to Paley. Nursing Philosophy doi:10 1111/nup 12068.

Scott, PA, Matthews, A \& Kirwan, M (2013) What is nursing in the $21^{\text {st }}$ century and what does the 21th century health system require of nursing? Nursing Philosophy 15, 23-34.

Seely, AJE (2013) Embracing the certainty of uncertainty: implications for health care and research. Perspectives in Biology and Medicine 56(1), 65-77

Shattell, M (2002) 'Eventually it 'Il be over': the dialectic between confinement and freedom in the world of the hospitalized patient In: Listening to patients $A$ phenomenological approach to nursing research and practice (Thomas, S P \& Pollio, H R eds) Springer Publishing Company, New York, 214-236. 
Shattell, M (2004) Nurse-patient interaction: a review of the literature. Journal of Clinical Nursing 6, 214-221.

Schell, JO \& O'Hare, AM (2013) Illness trajectories and their relevance to the care of adults with kidney disease. Current Opinion in Nephrology and Hypertension, 22(3), 316-324.

Siebens, K, Dierckx de Casterlé, B, Abraham, I, Dierckx, K, Braes, T, Darras, E, Dubois, M \& Milisen, K (2006) The professional self-image of nurses in Belgian hospitals : a crosssectional questionnaire survey. International Journal of Nursing Studies 43, 71-82.

Sorlie, V, Torjuul, K, Ross, A \& Kihlgren, M (2006) Satisfied patients are also vulnerable patients - narratives form an acute care ward. Journal of Clinical Nursing 15, 1240-1246.

Thorup, CB, Rundqvist, E, Roberts, C \& Delmar, C (2011) Care as a matter of courage: vulnerability, suffering and ethical formation in nursing care. Scandinavian Journal of Caring Sciences 26, 427-435.

Tichen, A (2000) Professional craft knowledge in patient-centered nursing and the facilitation of its development Doctoral dissertation, University of Oxford, UK .

Turkel, MC, Ray, MA \& Kornblatt, L (2012) Instead of reconceptualizing the nursing process let's re-name it. Nursing Science Quaterly 25(2), 194-198

Vanlaere, L \& Gastmans, C (2007) Ethics in nursing education: learning to reflect on care practices. Nursing Ethics 14(6), 758-766.

Vaismoradi, M, Salsali, M \& Ahmadi, F (2011) Nurses' experiences of uncertainty in clinical practice: a descriptive study. Journal of Advanced Nursing 67(5), 991-999.

Varcoe, C, Doane, G, Pauly, B, Rodney, P, Storch, J L, Mahoney, K, McPherson, G, Brown, H \& Starzomski, R (2004) Ethical practice in nursing: working the in-betweens. Journal of Advanced Nursing 45(3), 316-325.

Walker, AC (2002) Safety and comfort work of nurses glimpsed through patient narratives International Journal of Nursing Practice 1, 42-48. 
Wallerstedt, B \& Andershed, B (2007) Caring for dying patients outside special palliative care settings: experiences from a nursing perspective. Nordic College of Caring Science 21, $32-40$.

West, E, Barron, DN \& Reeves, R (2005) Overcoming the barriers to patient-centred care : time, tools and training. Journal of Clinical Nursing 14, 435-443.

Williams, AN (1998) The delivery of quality nursing care: a grounded theory study of the nurse's perspective. Journal of Advanced Nursing 27, 808-816.

Wysong, PR \& Driver, E (2009) Patients' perceptions of nurses' skills. Critical Care Nurse 29, 24-37. 